\title{
MULTI-MODAL IMAGE REGISTRATION USING ORDINAL FEATURES AND MULTI-DIMENSIONAL MUTUAL INFORMATION
}

\author{
Shu Liao and Albert C. S. Chung \\ Lo Kwee-Seong Medical Image Analysis Laboratory, \\ Department of Computer Science and Engineering, \\ The Hong Kong University of Science and Technology, Hong Kong.
}

\begin{abstract}
This paper proposes to use a new feature, namely ordinal feature, for image registration tasks. The ordinal features are extracted by passing through the images through the generalized ordinal filter bank, which effectively encodes the spatial information between neighboring voxels and specific microstructural information in the images. The ordinal feature is integrated with the image intensity to form a two-element attribute vector. Then, four dimensional mutual information is used as the similarity measure. The experimental results show that the proposed method is more robust than the conventional MI-based method and the method using Gabor features. The accuracy is comparable for the three approaches.
\end{abstract}

Index Terms - Image Registration, Ordinal Features, Multidimensional Mutual Information.

\section{INTRODUCTION}

Multi-modal image registration plays an important role in medical image analysis because complementary information can be obtained from images acquired by different modalities. The task of image registration is to reliably estimate the geometric transformation such that two images can be precisely aligned. Defining similarity measure has been a crucial part in image registration. Voxel intensity-based Mutual information (MI) $[1,2,3]$ is a widely used similarity measure in image registration tasks. However, it is also shown that the intensitybased MI can result in being trapped at the local maximum and thus lead to misalignment for some cases $[4,5]$. The disadvantage of the intensity-based MI is that it does not take the spatial information of voxel intensity distribution into consideration.

Therefore, we are motivated to propose a new feature, namely ordinal feature, for image registration task. The ordinal feature is extracted by using ordinal filters with different orders so as to represent the spatial information in the feature space. We will also show that the well-known Gabor filter is a special form of the ordinal filter. The ordinal feature is integrated with the image intensity to form a twoelement attribute vector to describe each voxel in the image.
Then, the multi-dimensional mutual information is defined as the similarity measure in the attribute space. The proposed method is evaluated by performing registration experiments on four MR-CT data sets obtained from the Retrospective Image Registration Evaluation project for studying both robustness and accuracy. It is experimentally shown that the proposed method consistently has higher success rate than the method using Gabor features and the conventional MI while the registration accuracy is comparable with the conventional MI and the 4D MI based on Gabor filter responses.

The paper is organized as follows. Section 2 introduces the concept of generalized ordinal filters and ordinal features. Section 3 formulates the multi-dimensional MI based on the ordinal features and the voxel intensities. Section 4 describes the optimization procedure. Section 5 analyzes the experimental results and Section 6 concludes the paper.

\section{ORDINAL FEATURES}

In this section, the ordinal features are introduced. The image spatial information can be reflected by the ordinal relationships among neighborhood voxels or regions in the images.

\subsection{Generalized Ordinal Filters}

The ordinal features are extracted by passing the input images through the ordinal filters. All the operations are performed in three dimensions for the registration experiments. However, for better illustration, we just show concepts in 2D cases. It can be easily extended to 3D. Gabor filter is a wellknown differential filter for the comparison of neighboring regions. Although Gabor filters are well-suited to encode local relationships between adjacent regions, they have significant disadvantages. Specifically, the span of the Gabor filter is limited by the size of its sub-fields, therefore it is difficult to capture useful information from small regions across large distances. Balas and Sinha [6] extended the Gabor filters to "dissociated dipoles" for non-local comparison to overcome such disadvantage, such filter consists of an excitatory lobe and an inhibitory lobe. 


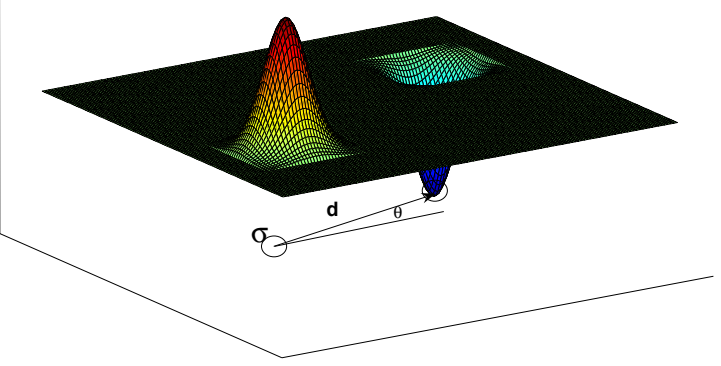

Fig. 1. The dissociated dipole filter.

There are three important parameters controlling the shape of the filter. (1) $\sigma$ is the parameter controlling the shape and scale of the lobe. If the lobes are selected as Gaussian filters, $\sigma$ is the standard deviation. (2) $d$ is defined as the distance between the centers of two lobes. It represents the targeting neighbor size. (3) $\theta$ controls the orientation of the filter. It denotes the angle between the horizontal axis and the line passing through the centers of two lobes. It ranges from 0 to $2 \pi$.

In this paper, the dissociated dipole filter is extended to the dissociated multi-poles ordinal filters. The number of lobes is denoted as the order of the ordinal filters. Figure 2 shows a 3 rd order and a 4 th order ordinal filter. The extended ordinal filter with multiple lobes can represent more complex microstructures than the dipole filter. Also, using different shapes of individual lobe, the ordinal filter can be designed to extract different specified micro-structures from the images. The Gabor filter is a special case of the ordinal filter.

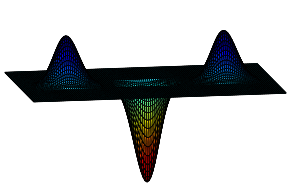

(a)

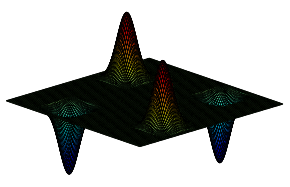

(b)
Fig. 2. (a) A 3rd order ordinal filter and (b) a 4th order ordinal filter.

In order to make the ordinal filter effective for brain image representation, there are three rules for the ordinal filter design:

1. Each individual lobe in the ordinal filter should be a low-pass filter in order to statistically estimate the spatial information within the region of each lobe.

2. The coefficients of the each individual lobe should be inversely proportional to its distance from the lobe center. The usage of this constraint is to maintain the locality of the filter. In this paper, the Gaussian mask is used as the lobe.

3. The coefficients of all lobes should be sum-to-zero so that the ordinal filter is not biased and the entropy of a single ordinal code is maximized. For example, in Figure 2, the sum of all excitatory lobe coefficients is equal to the sum of all the inhibitory lobe coefficients for each filter.

In this paper, 24 ordinal filters are used, as shown in Figure 3. The Gaussian parameter is set to $\sigma=\frac{\pi}{2}$. The inter-pole distances are $\mathrm{d}=8,12,16$, and 20 for the 2 nd order and 4 th order ordinal filters, and $\mathrm{d}=4,8,12$, and 16 for the 3rd order ordinal filters. For the 2 nd and 3 rd order ordinal filters, the orientations are 0 and $\frac{\pi}{2}$, for the 4 th order ordinal filters, the orientations are 0 and $\frac{\pi}{4}$.

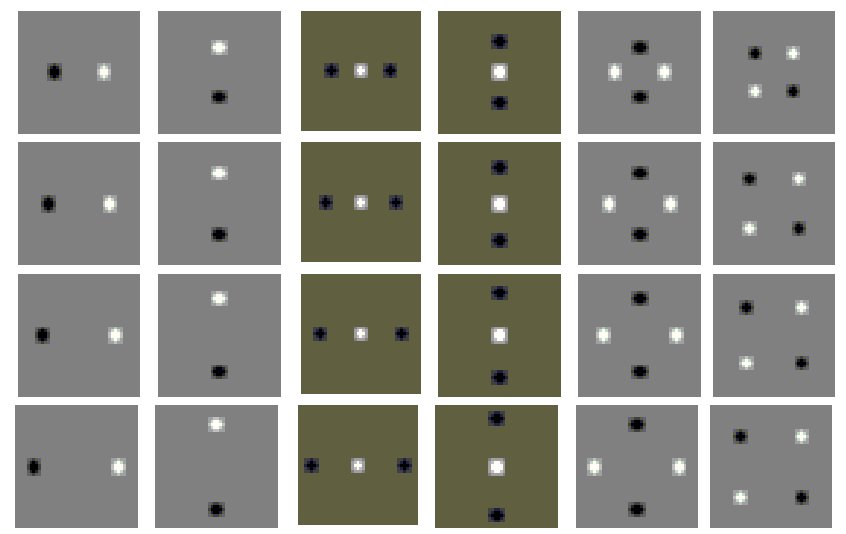

Fig. 3. The 24 ordinal filters used in the experiments.

\subsection{Construction of the Ordinal Feature Map and the Optimization}

After passing the images through the ordinal filter bank defined in Section 2.1, each voxel is now represented as a 24dimensional vector. However, for the current ordinal feature set, there is much redundant information. In order to remove such redundant information, K-Means clustering technique [7] is performed to form cluster centers in the feature space. In the experiments, the number of cluster centers was set to 
32. After the cluster centers are formed, each voxel is labeled by the cluster center, which is the closest center to its corresponding 24-dimensional vector so that the final ordinal feature map is formed.

\section{MULTI-DIMENSIONAL MUTUAL INFORMATION}

As we have discussed above, the ordinal feature map encodes the spatial information between neighboring voxels and the properties of specified micro-structures in the images. The feature is then associated with voxel intensity to form a twoelement attribute vector for registration. The multi-dimensional (4D, in this paper) mutual information (MI) is defined as the similarity measure.

Suppose $I_{r}$ and $I_{f}$ are the intensity domains of the reference and floating images respectively obtained from the same or different acquisitions. Their corresponding ordinal feature maps are denoted as $M_{r}$ and $M_{f}$. Given a rigid transformation $T$, the $4 \mathrm{D}$ joint histogram $h_{t}\left(I_{f}, M_{f}, I_{r}, M_{r}\right)$ over the sampling set $V^{3}$ is approximated by histogramming [8] in this paper because of computational efficiency. The trilinear partial volume interpolation [2] is adopted to achieve sub-voxel accuracy. The 4D mutual information is defined as,

$$
\operatorname{MI}-4 \mathrm{D}(T)=\sum_{I_{f}, M_{f}, I_{r}, M_{r}} A \cdot B,
$$

where $A$ is given as,

$$
A=p_{T}\left(I_{f}, M_{f}, I_{r}, M_{r}\right),
$$

and $B$ is defined as,

$$
B=\log _{2} \frac{p_{T}\left(I_{f}, M_{f}, I_{r}, M_{r}\right)}{p_{T}\left(I_{f}, M f\right) \cdot p_{T}\left(I_{r}, M_{r}\right)} .
$$

\section{OPTIMIZATION OF 4D-MI}

In the proposed approach, the optimal transformation $\hat{T}$ can be estimated by,

$$
\hat{T}=\arg \max _{T} \operatorname{MI}-4 \mathrm{D}(T) .
$$

The value of the 4D MI is maximized by using the Powell's method [9]. The Powell's method searches for the maximum value of $4 \mathrm{D}$ MI iteratively along each parameter axis $T$ while other parameters are kept constant. In the experiments, the search step $\partial \mathrm{T}$ was set to $0.02 \mathrm{~mm}$ for the translation parameters along $X, Y$ and $Z$ axes and 0.2 degrees for the rotation parameters about $X, Y$ and $Z$ axes.

\section{EXPERIMENTAL RESULTS}

To evaluate the performance of the proposed method in terms of the registration robustness and accuracy, a series of randomized experiments have been designed. The proposed method has also been compared with the conventional intensity-based MI method and the Gabor filter based method. For the Gabor filter based method, the process of generating the feature map is the same as the ordinal features, and the $4 \mathrm{D}$ MI is used as similarity measures. In this paper, the input images were passed through 16 Gabor filters with center frequencies: $F=2.00,3.17,5.04$ and 8.00 and oriented at angles of 0,45 , 90, 135 degrees to achieve optimal coverage in the Fourier domain. Four pairs of CT and MR image volumes were obtained from the Retrospective Image Registration Evaluation project. One of the pairs of 2D CT and MR image slices is shown in Figure 4. In this paper, the number of histogram bins are set to be 64 .
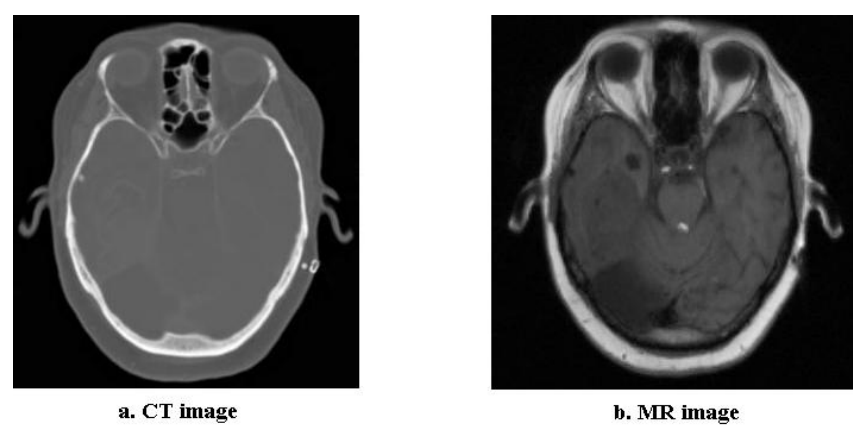

Fig. 4. One pair of $2 D C T$ and MR image slices.

In the experiments, the $\mathrm{CT}$ images were chosen as the reference images while MR images were chosen as the floating images. For each pre-obtained ground truth registration MRCT pairs, it was perturbed by six uniformly distributed random offsets for all translational and rotational axes. The perturbed image pairs were used as the starting alignment. For each MR-CT pair, the experiment was repeated 100 times. The random offsets for the translational parameters along $X$ and $Y$ axes were drawn between $[-150,150] \mathrm{mm}$, and between [-70,70] $\mathrm{mm}$ along $Z$ axis. The rotational random offsets for $X, Y$ and $Z$ axes were drawn between [-20,20] degrees. The root-sum-square of the differences for three translational axes was computed as the translation errors. The rotational errors were measured by the real part of the quaternion. The threshold vector for assessing registration success was set to $\left(2 \mathrm{~mm}, 2^{\circ}\right)$ because registration errors below $2 \mathrm{~mm}$ and $2^{\circ}$ are generally acceptable by experienced clinicians $[10,11]$. The registration success rates for the proposed method, conventional MI and the Gabor filter based method for the aforementioned four MR-CT datasets (\#1, \#2, \#3 and \#4) are listed in Table 1

As it is shown in the table, the proposed method has higher success rate than the conventional intensity based MI and the Gabor feature based approach as the ordinal features embed spatial information and micro-structural characteristics of the images and it has the advantage of capturing useful informa- 


\begin{tabular}{|c|c|c|c|}
\hline $\begin{array}{c}\text { Testing } \\
\text { dataset }\end{array}$ & $\begin{array}{c}\text { MI } \\
\text { SR }\end{array}$ & $\begin{array}{c}\text { Gabor Features, 4D-MI } \\
\text { SR }\end{array}$ & $\begin{array}{c}\text { Ordinal Features, 4D-MI } \\
\text { SR }\end{array}$ \\
\hline$\# 1$ & $68 \%$ & $74 \%$ & $84 \%$ \\
$\# 2$ & $61 \%$ & $83 \%$ & $90 \%$ \\
$\# 3$ & $65 \%$ & $81 \%$ & $92 \%$ \\
$\# 4$ & $74 \%$ & $78 \%$ & $83 \%$ \\
\hline
\end{tabular}

\section{CONCLUSION}

In this paper, a new feature, called ordinal features, is proposed for multi-modal image registration task. The ordinal filter is more general than the Gabor filter as it can capture local information from different regions across large scales. The

Table 1. Success rates of the conventional MI, Gabor Feature based approach and the proposed approach $. S R=$ Success Rates.

tion from small regions across different scales over the Gabor filters.

To further compare the registration accuracy among the proposed method, Gabor feature based method and the conventional intensity based MI approach, the mean value and standard deviation of the registration errors in the successful registrations in all four MR-CT pairs for both methods were calculated, as listed in Tables 2 and 3.

\begin{tabular}{|c|ccc|}
\hline Method & \multicolumn{3}{|c|}{ Translation $\left(10^{-3} m m\right)$} \\
& $\Delta t_{x}$ & $\Delta t_{y}$ & $\Delta t_{z}$ \\
\hline MI & $0.82 \pm 0.86$ & $1.03 \pm 0.38$ & $-0.82 \pm 0.73$ \\
Gabor Features, 4D-MI & $0.65 \pm 0.96$ & $-1.15 \pm 0.22$ & $-1.03 \pm 0.88$ \\
Ordinal Features, 4D-MI & $1.15 \pm 0.62$ & $0.95 \pm 0.57$ & $-1.25 \pm 0.40$ \\
\hline
\end{tabular}

Table 2. Registration accuracy in translation for the conventional MI approach, Gabor feature based method and the proposed approach.

\begin{tabular}{|c|ccc|}
\hline Method & \multicolumn{3}{|c|}{ Translation (10 $0^{-3}$ degree $)$} \\
& $\Delta \theta_{x}$ & $\Delta \theta_{y}$ & $\Delta \theta_{z}$ \\
\hline MI & $-0.75 \pm 0.82$ & $0.74 \pm 1.13$ & $0.93 \pm 1.37$ \\
Gabor Features, 4D-MI & $-0.91 \pm 1.06$ & $-0.42 \pm 0.87$ & $1.21 \pm 0.79$ \\
Ordinal Features, 4D-MI & $-1.16 \pm 0.80$ & $0.63 \pm 1.08$ & $0.77 \pm 1.41$ \\
\hline
\end{tabular}

Table 3. Registration accuracy in rotation for the conventional MI approach, Gabor feature based method and the proposed approach.

Tables 2 and 3 show that the registration accuracy of the proposed method is comparable with the Gabor feature based method and the conventional intensity based MI method. However, we should bear in mind that the number of the successful registrations of the proposed method is significantly higher than the Gabor feature based method and the conventional intensity-based MI method as illustrated in Table 1. It is also shown that the registration accuracy of the proposed method can reach the precise level of $10^{-3} \mathrm{~mm}$ in average for the successful registration cases, thought the $2 \mathrm{~mm}$ threshold is used.

The computation time for the proposed method is about 3 minutes for each testing case, compared with the running time of about 1 minute for the conventional MI method. ordinal feature map of an input image is obtained by passing the image through the designed ordinal filter bank, performing K-Means clustering, and finally assigning the voxel with the label of the closest cluster center. In this paper, the 2nd, 3rd and 4th order ordinal filters are used as the filter bank, and the Gaussian mask is used as the individual lobe. Then, the 4D MI is exploited as the similarity measure by integrating the ordinal feature map with the voxel intensity for rigid registration. Experimental results show that the proposed method has higher success rate than the Gabor feature based method and the conventional MI method while the registration accuracy is comparable with the two compared methods. The future directions are that the shape of the individual lobe for each filter can be further extended to better fit the registration task, and higher order ordinal filters can also be adopted.

\section{REFERENCES}

[1] W.M. Wells, P. Viola, and et al., "Multi-Modal Volume Registration by Maximization of Mutual Information," Medical Image Analysis, vol. 1, no. 1, pp. 35-51, 1996.

[2] F. Maes, A. Collignon, and et al., "Multimodality Image Registration by Maximization of Mutual Information," TMI, vol. 16, no. 2, pp. 187198, 1997.

[3] J.P.W. Pluim, J.B.A. Maintz, and M.A. Viergever, "Mutualinformation-based registration of medical images: a survey," $T M I$, vol. 22, no. 8, pp. 986-1004, 2003.

[4] G. Penney, J. Weese, and et al., "A Comparison of Similarity Measures for Use in 2D-3D Medical Image Registration," TMI, vol. 17, pp. 586595, 1998.

[5] J.P.W. Pluim, J.B.A. Maintz, and M.A. Viergever, "Image Registration by Maximization of Combined Mutual Information and Gradient Information," TMI, vol. 19, pp. 809-814, 2000.

[6] B. Balas and P. Sinha, "Dissociated dipoles: Image representation via non-local comparisons," in CBCL Paper \#229/AI Memo \#2003-018, MIT Computer Science and Artificial Intelligence Laboratory, 2003.

[7] J. A. Hartigan and M. A. Wong, "A k-means clustering algorithm," Applied Statistics, vol. 28, no. 1, pp. 100-108, 1979.

[8] C.M. Bishop, Neural Networks for Pattern Recognition, Oxford U. Press, 1995.

[9] W.H. Press, S.A. Teukolsky, and et al., Numerical Recipes in C, Cambridge University Press, 1992.

[10] J.V. Hajnal, D.L.G. Hill, and D.J. Hawkes, Medical Image Registration, CRC Press LLC, 2001.

[11] Y. Zhu and S. Cochoff, "Likelihood Maximization Approach to Image Registration," TIP, vol. 11, pp. 1417-1426, 2002. 